DOI: http://dx.doi.org/10.18273/revint.v36n1-2018004

\title{
A brief description of operators associated to the quantum harmonic oscillator on Schatten-von Neumann classes
}

\author{
Duván CARDOnA* \\ Pontificia Universidad Javeriana, Mathematics Department, Bogotá, Colombia
}

\begin{abstract}
In this note we study pseudo-multipliers associated to the harmonic oscillator (also called Hermite multipliers) belonging to Schatten classes on $L^{2}\left(\mathbb{R}^{n}\right)$. We also investigate the spectral trace of these operators. Keywords: Harmonic oscillator, Fourier multiplier, Hermite multiplier, nuclear operator, traces.
\end{abstract}

MSC2010: 81Q10, 47B10, 81Q05.

\section{Una descripción breve de operadores asociados al oscilador armónico cuántico sobre las clases de Schatten-von Neumann}

Resumen. En esta nota se estudia una clase de operadores definidos a través del espectro del oscilador armónico y conocidos en la literatura como pseudo multiplicadores (pseudo multiplicadores de Hermite). Se analizan criterios óptimos para clasificar estos operadores en las clases de Schatten-von Neumann sobre $L^{2}\left(\mathbb{R}^{n}\right)$. El trabajo culmina con una investigación sobre la traza espectral y/o nuclear de tales operadores.

Palabras clave: Oscilador armónico, multiplicador de Fourier, multiplicadores de Hermite, operador nuclear, trazas.

\section{Introduction}

\subsection{Outline of the paper}

Pseudo-multipliers and multipliers associated to the harmonic oscillator arise from the study of Hermite expansions for complex functions on $\mathbb{R}^{n}$ (see Thangavelu [23], [24],

\footnotetext{
${ }^{*}$ E-mail: cardonaduvan $₫ j$ averiana.edu.co

Received: 20 March 2018, Accepted: 22 June 2018.

To cite this article: D. Cardona, A brief description of operators associated to the quantum harmonic oscillator on Schatten-von Neumann classes, Rev. Integr. temas mat. 36 (2018), No. 1, 49-57. doi: 10.18273/revint.v36n1-2018004
} 
[25], [26] [27], [28], Epperson [11] and Bagchi and Thangavelu [1]). At the same time, we note that pseudo-multipliers are pseudo-differential operators on $\mathbb{R}^{n}$ in view of the quantization process developed by Ruzhansky and Tokmagambetov in [17] and [18] when the reference operator is the harmonic oscillator. In this note, we are interested in the membership of pseudo-multipliers associated to the harmonic oscillator (also called Hermite pseudo-multipliers) in the Schatten classes, $S_{r}\left(L^{2}\right)$ on $L^{2}\left(\mathbb{R}^{n}\right)$. With this paper we finish the classification of pseudo-multipliers in classes of $r$-nuclear operators on $L^{p_{-}}$ spaces (see Barraza and Cardona [2], [3]), which on $L^{2}\left(\mathbb{R}^{n}\right)$ coincide with the Schattenvon Neumann classes of order $r$. Our main result is Theorem 1.1 where we establish some criteria in order that pseudo-multipliers belong to the classes $S_{r}\left(L^{2}\right), 0<r \leq 2$. In order to present our main result we recall some notions. Let us consider the sequence of Hermite functions on $\mathbb{R}^{n}$,

$$
\phi_{\nu}=\Pi_{j=1}^{n} \phi_{\nu_{j}}, \quad \phi_{\nu_{j}}\left(x_{j}\right)=\left(2^{\nu_{j}} \nu_{j} ! \sqrt{\pi}\right)^{-\frac{1}{2}} H_{\nu_{j}}\left(x_{j}\right) e^{-\frac{1}{2} x_{j}^{2}}
$$

where $x=\left(x_{1}, \cdots, x_{n}\right) \in \mathbb{R}^{n}, \nu=\left(\nu_{1}, \cdots, \nu_{n}\right) \in \mathbb{N}_{0}^{n}$, and $H_{\nu_{j}}\left(x_{j}\right)$ denotes the Hermite polynomial of order $\nu_{j}$. It is well known that the Hermite functions provide a complete and orthonormal system in $L^{2}\left(\mathbb{R}^{n}\right)$. If we consider the operator $L=-\Delta+|x|^{2}$ acting on the Schwartz space $\mathscr{S}\left(\mathbb{R}^{n}\right)$, where $\Delta$ is the standard Laplace operator on $\mathbb{R}^{n}$, then we have the relation $L \phi_{\nu}=\lambda_{\nu} \phi_{\nu}, \nu \in \mathbb{N}_{0}^{n}$. The operator $L$ is symmetric and positive in $L^{2}\left(\mathbb{R}^{n}\right)$ and admits a self-adjoint extension $H$ whose domain is given by

$$
\operatorname{Dom}(H)=\left\{\sum_{\nu \in \mathbb{N}_{0}^{n}}\left\langle f, \phi_{\nu}\right\rangle_{L^{2}} \phi_{\nu}: \sum_{\nu \in \mathbb{N}_{0}^{n}}\left|\lambda_{\nu}\left\langle f, \phi_{\nu}\right\rangle_{L^{2}}\right|^{2}<\infty\right\} .
$$

So, for $f \in \operatorname{Dom}(H)$, we have

$$
(H f)(x)=\sum_{\nu \in \mathbb{N}_{0}} \lambda_{\nu} \widehat{f}\left(\phi_{\nu}\right) \phi_{\nu}(x), \widehat{f}\left(\phi_{\nu}\right)=\left\langle f, \phi_{\nu}\right\rangle_{L^{2}}
$$

The operator $H$ is precisely the quantum harmonic oscillator on $\mathbb{R}^{n}$ (see [15]). The sequence $\left\{\widehat{f}\left(\phi_{v}\right)\right\}$ determines the Fourier-Hermite transform of $f$, with corresponding inversion formula

$$
f(x)=\sum_{\nu \in \mathbb{N}_{0}^{n}} \widehat{f}\left(\phi_{v}\right) \phi_{\nu}(x) .
$$

On the other hand, pseudo-multipliers are defined by the quantization process that associates to a function $m$ on $\mathbb{R}^{n} \times \mathbb{N}_{0}^{n}$ a linear operator $T_{m}$ of the form

$$
T_{m} f(x)=\sum_{\nu \in \mathbb{N}_{0}^{n}} m(x, \nu) \widehat{f}\left(\phi_{\nu}\right) \phi_{\nu}(x), \quad f \in \operatorname{Dom}\left(T_{m}\right) .
$$

The function $m$ on $\mathbb{R}^{n} \times \mathbb{N}_{0}^{n}$ is called the symbol of the pseudo-multiplier $T_{m}$. If in (5), $m(x, \nu)=m(\nu)$ for all $x$, the operator $T_{m}$ is called a multiplier. Multipliers and pseudomultipliers have been studied, for example, in the works [1], [20], [21], [22], [23], [24] (and references therein) principally by its mapping properties on $L^{p}$ spaces. In order that the operator $T_{m}: L^{2}\left(\mathbb{R}^{n}\right) \rightarrow L^{2}\left(\mathbb{R}^{n}\right)$ belongs to the Schatten class $S_{r}\left(L^{2}\right)$, in this paper we provide some (sharp) conditions on the symbol $m$.

[Revista Integración, temas de matemáticas 


\subsection{Pseudo-multipliers in Schatten classes}

By following A. Grothendieck [12], we can recall that a linear operator $T: E \rightarrow F(E$ and $F$ Banach spaces) is $r$-nuclear, if there exist sequences $\left(e_{n}^{\prime}\right)_{n \in \mathbb{N}_{0}}$ in $E^{\prime}$ (the dual space of $E$ ) and $\left(y_{n}\right)_{n \in \mathbb{N}_{0}}$ in $F$ such that

$$
T f=\sum_{n \in \mathbb{N}_{0}} e_{n}^{\prime}(f) y_{n}, \quad \text { and } \quad \sum_{n \in \mathbb{N}_{0}}\left\|e_{n}^{\prime}\right\|_{E^{\prime}}^{r}\left\|y_{n}\right\|_{F}^{r}<\infty .
$$

The class of $r$-nuclear operators is usually endowed with the quasi-norm

$$
n_{r}(T):=\inf \left\{\left\{\sum_{n}\left\|e_{n}^{\prime}\right\|_{E^{\prime}}^{r}\left\|y_{n}\right\|_{F}^{r}\right\}^{\frac{1}{r}}: T=\sum_{n} e_{n}^{\prime} \otimes y_{n}\right\} .
$$

In addition, when $E=F$ is a Hilbert space and $r=1$ (resp. $r=2$ ), the definition above agrees with the concept of trace class operators (resp. Hilbert-Schmidt). For the case of Hilbert spaces $H$, the set of $r$-nuclear operators agrees with the Schatten-von Neumann class of order $r$ (see Pietsch [13], [14]). We recall that a linear operator $T$ on a Hilbert space $H$ belong to the Schatten class of order $r, S_{r}(H)$, if

$$
s_{r}(T):=\sum_{n \in \mathbb{N}_{0}} \lambda_{n}(T)^{r}<\infty,
$$

where $\left\{\lambda_{n}(T)\right\}$ denotes the sequence of singular values of $T$, which are the eigenvalues of the operator $\sqrt{T^{*} T}$. It was proved in [2] that a multiplier $T_{m}$, with symbol satisfying conditions of the form

$$
\varkappa\left(m, p_{1}, p_{2}\right):=\sum_{s=0}^{n} \sum_{\nu \in I_{s}} \alpha_{r, p_{1}, p_{2}}(s, \nu)|m(\nu)|^{r}<\infty
$$

where $\left\{I_{s}\right\}_{s=0}^{n}$ is a suitable partition of $\mathbb{N}_{0}^{n}$, and $\alpha_{r, p_{1}, p_{2}}(s, \nu)$ is a suitable kernel, can be extended to a $r$-nuclear operator from $L^{p_{1}}\left(\mathbb{R}^{n}\right)$ into $L^{p_{2}}\left(\mathbb{R}^{n}\right)$. Although is easy to see that similar necessary conditions apply for pseudo-multipliers, the $r$-nuclearity for these operators in $L^{p}$-spaces was characterized in [3] by the following condition:

- a pseudo-multiplier $T_{m}$ can be extended to a $r$-nuclear operator from $L^{p_{1}}$ into $L^{p_{2}}$ if and only if there exist functions $h_{k}$ and $g_{k}$ satisfying

$$
m(x, \nu)=\phi_{\nu}(x)^{-1} \sum_{k=1}^{\infty} h_{k}(x) \widehat{g}\left(\phi_{\nu}\right), \quad \phi_{\nu}(x) \neq 0, \text { with } \sum_{k=0}^{\infty}\left\|g_{k}\right\|_{L^{p_{1}^{\prime}}}^{r}\left\|h_{k}\right\|_{L^{p_{2}}}^{r}<\infty .
$$

If we consider $p_{1}=p_{2}=2$, and a multiplier $T_{m}$, the conditions above can be replaced by the following more simple one,

$$
\varkappa(m, 2, r):=\sum_{\nu \in \mathbb{N}_{0}}|m(\nu)|^{r}<\infty,
$$

Vol. 36, $\left.\mathrm{N}^{\circ} 1,2018\right]$ 
because the set of singular values of a multiplier $T_{m}$ consists of the elements in the sequence $\{|m(\nu)|\}_{\nu \in \mathbb{N}_{0}^{n}}$. The condition (10) characterizes the membership of pseudomultipliers in Schatten classes in terms of the existence of certain measurable functions. However, in this paper we provide explicit conditions on $m$ in order to guarantee that $T_{m} \in S_{r}\left(L^{2}\right)$, because explicit conditions allow us to known information about the distribution of the spectrum of these operators. Our main result is the following theorem.

Theorem 1.1. Let $T_{m}$ be a pseudo-multiplier with symbol $m$ defined on $\mathbb{R}^{n} \times \mathbb{N}_{0}^{n}$. Then we have:

- $T_{m}$ is a Hilbert-Schmidt operator on $L^{2}\left(\mathbb{R}^{n}\right)$, i.e., $T_{m} \in S_{2}\left(L^{2}\right)$, if and only if

$$
\sum_{\nu \in \mathbb{N}_{0}^{n}} \int_{\mathbb{R}^{n}}|m(x, \nu)|^{2} \phi_{\nu}(x)^{2} d x<\infty
$$

- If $T_{m}$ is a positive operator, then $T_{m}$ is trace class, i.e., $T_{m} \in S_{1}\left(L^{2}\right)$, if and only if

$$
\sum_{\nu \in \mathbb{N}_{0}^{n}} \int_{\mathbb{R}^{n}} m(x, \nu) \phi_{\nu}(x)^{2} d x<\infty
$$

- $T_{m} \in S_{r}\left(L^{2}\right), 0<r \leq 1$, if

$$
\sum_{\nu \in \mathbb{N}_{0}^{n}}\left(\int_{\mathbb{R}^{n}}|m(x, \nu)|^{2} \phi_{\nu}(x)^{2} d x\right)^{\frac{r}{2}}<\infty .
$$

- If $1<r<2$ and there exists $\sigma>n\left(\frac{1}{r}-\frac{1}{2}\right)$ such that

$$
\sum_{\nu \in \mathbb{N}_{0}^{n}}|\nu|^{2 \sigma} \int_{\mathbb{R}^{n}}|m(x, \nu)|^{2} \phi_{\nu}(x)^{2} d x<\infty
$$

then $T_{m} \in S_{r}\left(L^{2}\right)$.

In general, on a Banach space compact linear operators are bounded operators. Taking into account that Schatten-von Neumann classes on Hilbert spaces are families of compact operators, our main theorem gives conditions for the $L^{2}\left(\mathbb{R}^{n}\right)$-continuity of pseudomultipliers. The problem of finding "satisfactory" conditions for the $L^{2}\left(\mathbb{R}^{n}\right)$-boundedness of pseudo-multipliers remains open, and it was proposed by Bagchi and Thangavelu in [1]; with our main result and the conditions proposed in Cardona and Barraza [3], we solve partially such problem. However, Bagchi-Thangavelu's problem will be "satisfactorily" solved in the work Cardona and Ruzhansky [4].

\subsection{Related works}

Now, we include some references on the subject. Sufficient conditions for the $r$-nuclearity of spectral multipliers associated to the harmonic oscillator, but in modulation spaces and Wiener amalgam spaces, have been considered by J. Delgado, M. Ruzhansky and B.

[Revista Integración, temas de matemáticas 
Wang in [8], [9]. The Properties of these multipliers in $L^{p}$-spaces have been investigated in the references S. Bagchi, S. Thangavelu [1], J. Epperson [11], K. Stempak and J.L. Torrea [20], [21], [22], S. Thangavelu [23], [24] and references therein. Hermite expansions for distributions can be found in B. Simon [19]. The $r$-nuclearity and Grothendieck-Lidskii formulae for multipliers and other types of integral operators can be found in [7], [9]. On Hilbert spaces the class of $r$-nuclear operators agrees with the Schatten-von Neumann class $S_{r}(H)$; in this context operators with integral kernel on Lebesgue spaces and, in particular, operators with kernel acting of a special way with anharmonic oscillators of the form $E_{a}=-\Delta_{x}+|x|^{a}, a>0$, has been considered on Schatten classes on $L^{2}\left(\mathbb{R}^{n}\right)$ in J. Delgado and M. Ruzhansky [10]. A complete treatment for $L^{p}$-boundedness and $L^{p}$-compactness properties in terms of the Littlewood-Paley theory and the Hörmander condition will be considered in Cardona and Ruzhansky [4]. The proof of our results will be presented in the next section.

\section{Pseudo-multipliers in Schatten-von Neumann classes}

In this section we prove our main result for pseudo-multipliers $T_{m}$. Our criteria will be formulated in terms of the symbols $m$. First, let us observe that every pseudo-multiplier $T_{m}$ is an operator with kernel $K_{m}(x, y)$. In fact, straightforward computation shows that

$$
T_{m} f(x)=\int_{\mathbb{R}^{n}} K_{m}(x, y) f(y) d y, K_{m}(x, y):=\sum_{\nu \in \mathbb{N}_{0}^{n}} m(x, \nu) \phi_{\nu}(x) \phi_{\nu}(y)
$$

for every $f \in \mathscr{D}\left(\mathbb{R}^{n}\right)$. We will use the following result (see J. Delgado [5], [6]).

Theorem 2.1. Let us consider $1 \leq p_{1}, p_{2}<\infty, 0<r \leq 1$ and let $q_{i}$ be such that $\frac{1}{p_{i}}+\frac{1}{q_{i}}=1$. Let $\left(X_{1}, \mu_{1}\right)$ and $\left(X_{2}, \mu_{2}\right)$ be $\sigma$-finite measure spaces. An operator $T$ : $L^{p_{1}}\left(X_{1}, \mu_{1}\right) \rightarrow L^{p_{2}}\left(X_{2}, \mu_{2}\right)$ is $r$-nuclear if and only if there exist sequences $\left(g_{n}\right)_{n}$ in $L^{p_{2}}\left(\mu_{2}\right)$, and $\left(h_{n}\right)$ in $L^{q_{1}}\left(\mu_{1}\right)$, such that

$$
\sum_{n}\left\|g_{n}\right\|_{L^{p_{2}}}^{r}\left\|h_{n}\right\|_{L^{q_{1}}}^{r}<\infty, \text { and } T f(x)=\int\left(\sum_{n} g_{n}(x) h_{n}(y)\right) f(y) d \mu_{1}(y), \text { a.e.w. } x,
$$

for every $f \in L^{p_{1}}\left(\mu_{1}\right)$. In this case, if $p_{1}=p_{2}$ (see Section 3 of [5]) the nuclear trace of $T$ is given by

$$
\operatorname{Tr}(T):=\int \sum_{n} g_{n}(x) h_{n}(x) d \mu_{1}(x)
$$

Now, we prove our main theorem.

Proof of Theorem 1.1. Let us consider a pseudo-multiplier $T_{m}$. By definition, $T_{m}$ is a Hilbert-Schmidt operator if and only if there exists an orthonormal basis $\left\{e_{\nu}\right\}_{\nu}$ of $L^{2}\left(\mathbb{R}^{n}\right)$ such that

$$
\sum_{\nu}\left\|T_{m} e_{\nu}\right\|_{L^{2}}^{2}<\infty
$$

In particular, if we choose the system of Hermite functions $\left\{\phi_{\nu}\right\}$, which provides an orthonormal basis of $L^{2}\left(\mathbb{R}^{n}\right)$, from the relation $T_{m}\left(\phi_{\nu}\right)=m(x, \nu) \phi_{\nu}$ we conclude that

Vol. 36, $\left.\mathrm{N}^{\circ} 1,2018\right]$ 
$T_{m}$ is of Hilbert-Schmidt type, if and only if

$$
\sum_{\nu}\left\|m(\cdot, \nu) \phi_{\nu}\right\|_{L^{2}}^{2}=\sum_{\nu \in \mathbb{N}_{0}^{n}} \int_{\mathbb{R}^{n}}|m(x, \nu)|^{2} \phi_{\nu}(x)^{2} d x<\infty .
$$

So, we have proved the first statement. Now, if we assume that $T_{m}$ is positive, then $T_{m}$ is of class trace if and only if there exists an orthonormal basis $\left\{e_{\nu}\right\}_{\nu}$ of $L^{2}\left(\mathbb{R}^{n}\right)$ such that

$$
\sum_{\nu}\left\langle T_{m} e_{\nu}, e_{\nu}\right\rangle_{L^{2}}<\infty
$$

As in the first assertion, if we choose the basis formed by the Hermite functions, $T_{m}$ is of class trace if and only if

$$
\sum_{\nu}\left\langle T_{m} e_{\nu}, e_{\nu}\right\rangle_{L^{2}}=\sum_{\nu \in \mathbb{N}_{0}^{n}} \int_{\mathbb{R}^{n}} m(x, \nu) \phi_{\nu}(x)^{2} d x<\infty,
$$

which proves the second assertion. Now, we will verify that (14) implies that $T_{m} \in$ $S_{r}\left(L^{2}\right)$ for $0<r \leq 1$. For this, we will use Delgado's Theorem (Theorem 2.1) to the representation (16) of $K_{m}$,

$$
K_{m}(x, y):=\sum_{\nu \in \mathbb{N}_{0}^{n}} m(x, \nu) \phi_{\nu}(x) \phi_{\nu}(y)
$$

So, $T_{m} \in S_{r}\left(L^{2}\right)$ if

$$
\sum_{\nu}\|m(\cdot, \nu)\|_{L^{2}}^{r}\left\|\phi_{\nu}\right\|_{L^{2}}^{r}=\sum_{\nu \in \mathbb{N}_{0}^{n}}\left(\int_{\mathbb{R}^{n}}|m(x, \nu)|^{2} \phi_{\nu}(x)^{2} d x\right)^{\frac{r}{2}}<\infty,
$$

where we have used that the $L^{2}$-norm of every Hermite function $\phi_{\nu}$ is normalised. In order to finish the proof, we only need to prove that (15) assures that $T_{m} \in S_{r}\left(L^{2}\right)$ for $1<r<2$. This can be proved by using the following multiplication property on Schatten classes:

$$
S_{p}(H) S_{q}(H) \subset S_{r}(H), \frac{1}{r}=\frac{1}{p}+\frac{1}{q} .
$$

So, we will factorize $T_{m}$ as

$$
T_{m}=T_{m} H^{\sigma} H^{-\sigma}, \sigma>0,
$$

where $H$ is the harmonic oscillator. Let us note that the symbol of $A=T_{m} H^{\sigma}$ is given by $a(x, \nu)=m(x, \nu)(2|\nu|+n)^{\sigma}$. So, from the first assertion, $A \in S_{2}\left(L^{2}\right)$ if and only if

$$
\sum_{\nu \in \mathbb{N}_{0}^{n}}|\nu|^{2 \sigma} \int_{\mathbb{R}^{n}}|m(x, \nu)|^{2} \phi_{\nu}(x)^{2} d x \asymp \sum_{\nu \in \mathbb{N}_{0}^{n}}(2|\nu|+n)^{2 \sigma} \int_{\mathbb{R}^{n}}|m(x, \nu)|^{2} \phi_{\nu}(x)^{2} d x<\infty .
$$

In order to prove that $T_{m} \in S_{r}\left(L^{2}\right)$, in view of the multiplication property

$$
S_{2}\left(L^{2}\right) S_{\frac{2 r}{2-r}}\left(L^{2}\right) \subset S_{r}\left(L^{2}\right), \frac{1}{r}=\frac{1}{2 r /(2-r)}+\frac{1}{2},
$$


we only need to prove that $H^{-\sigma} \in S_{p}\left(L^{2}\right)$ with $p=\frac{2 r}{2-r}$. The symbol of $H^{-\sigma}$ is given by $a^{\prime}(\nu)=(2|\nu|+n)^{-\sigma}$. By using the hypothesis $\sigma>n\left(\frac{1}{r}-\frac{1}{2}\right)$ we have that

$$
\sum_{\nu}\left|a^{\prime}(\nu)\right|^{p}=\sum_{\nu}(2|\nu|+n)^{-\sigma p}<\infty
$$

because $\sigma p=\sigma\left(\frac{1}{r}-\frac{1}{2}\right)^{-1}>n$. So, we finish the proof.

\subsection{Trace class pseudo-multipliers of the harmonic oscillator}

In order to determinate a relation with the eigenvalues of $T_{m}$ we recall the following result (see [16]).

Theorem 2.2. Let $T: L^{p}(\mu) \rightarrow L^{p}(\mu)$ be a r-nuclear operator as in $(6)$. If $\frac{1}{r}=1+\left|\frac{1}{p}-\frac{1}{2}\right|$, then,

$$
\operatorname{Tr}(T):=\sum_{n \in \mathbb{N}_{0}^{n}} e_{n}^{\prime}\left(f_{n}\right)=\sum_{n} \lambda_{n}(T),
$$

where $\lambda_{n}(T), n \in \mathbb{N}$, is the sequence of eigenvalues of $T$ with multiplicities taken into account.

As an immediate consequence of the preceding theorem (or the classical GrothendieckLidskii Theorem), if $T_{m}: L^{2}\left(\mathbb{R}^{n}\right) \rightarrow L^{2}\left(\mathbb{R}^{n}\right)$ is trace class (1-nuclear) then,

$$
\operatorname{Tr}\left(T_{m}\right)=\int_{\mathbb{R}^{n}} \sum_{\nu \in \mathbb{N}_{0}^{n}} m(x, \nu) \phi_{\nu}(x)^{2} d x=\sum_{n} \lambda_{n}(T),
$$

where $\lambda_{n}(T), n \in \mathbb{N}$, is the sequence of eigenvalues of $T_{m}$ with multiplicities taken into account.

\section{Acknowledgements}

The author would like to thank the anonymous referee for his careful evaluation and suggestions. We also thanks to Michael Ruzhansky for several discussions on the subject. This work was supported by the Department of Mathematics of the Pontificia Universidad Javeriana.

\section{References}

[1] Bagchi S. and Thangavelu S., "On Hermite pseudo-multipliers", J. Funct. Anal. 268 (2015), No. 1, 140-170,

[2] Barraza E.S. and Cardona D., "On nuclear $L^{p}$-multipliers associated to the Harmonic oscillator", in Analysis in Developing Countries, Springer Proceedings in Mathematics \& Statistics, Springer (2018), M. Ruzhansky and J. Delgado (Eds), to appear.

[3] Cardona D. and Barraza E.S., "Characterization of nuclear pseudo-multipliers associated to the harmonic oscillator", to appear in, Politehn. Univ. Bucharest Sci. Bull. Ser. A Appl. Math. Phys. (2018), arXiv:1709.07961.

Vol. 36, $\left.\mathrm{N}^{\circ} 1,2018\right]$ 
[4] Cardona D. and Ruzhansky M., "Hörmander condition for pseudo-multipliers associated to the harmonic oscillator", preprint.

[5] A trace formula for nuclear operators on $L^{p}$, in Pseudo-Differential Operators: Complex Analysis and Partial Differential Equations, Operator Theory: Advances and Applications 205, Schulze, B.W., Wong, M.W. (eds.), Birkhäuser, Basel (2010), 181-193.

[6] Delgado J., "The trace of nuclear operators on $L^{p}(\mu)$ for $\sigma$-finite Borel measures on second countable spaces", Integr. Equ. Oper. Theory 68 (2010), No- 1, 61-74.

[7] Delgado J., "On the $r$-nuclearity of some integral operators on Lebesgue spaces", Tohoku Math. J. (2) 67 (2015), No. 1, 125-135.

[8] Delgado J., Ruzhansky M. and Wang B., "Approximation property and nuclearity on mixednorm $L^{p}$, modulation and Wiener amalgam spaces", J. Lond. Math. Soc.(2) 94 (2016), 391-408.

[9] Delgado J., Ruzhansky M. and Wang B., "Grothendieck-Lidskii trace formula for mixednorm $L^{p}$ and variable Lebesgue spaces", to appear in J. Spectr. Theory, arXiv:1604.00198.

[10] Delgado J. and Ruzhansky M., "Schatten-von Neumann classes of integral operators", arXiv:1709.06446.

[11] Epperson J., "Hermite multipliers and pseudo-multipliers", Proc. Amer. Math. Soc. 124 (1996), No. 7, 2061-2068.

[12] Grothendieck A., "Produits tensoriels topologiques et espaces nucléaires", in: Mem. Amer. Math. Soc. 16, Providence, 1955.

[13] Pietsch A., Operator ideals, Mathematische Monographien 16, VEB Deutscher Verlag der Wissenschaften, Berlin, 1978.

[14] Pietsch A., History of Banach spaces and linear operators, Birkhäuser Boston Inc., Boston, 2007.

[15] Prugovečki E., Quantum mechanics in Hilbert space, Pure and Applied Mathematics 92, Academic Press Inc., New York-London, 1981.

[16] Reinov O.I. and Latif Q., "Grothendieck-Lidskii theorem for subspaces of Lp-spaces", Math. Nachr. 286 (2013), No. 2-3, 279-282.

[17] Ruzhansky M. and Tokmagambetov N., "Nonharmonic analysis of boundary value problems", Int. Math. Res. Notices 12 (2016), 3548-3615.

[18] Ruzhansky M. and Tokmagambetov N., "Nonharmonic analysis of boundary value problems without WZ condition", Math. Model. Nat. Phenom. 12 (2017), No. 1, 115-140.

[19] Simon B., "Distributions and their Hermite expansions", J. Math. Phys. 12 (1971), No. 1, $140-148$.

[20] Stempak K., "Multipliers for eigenfunction expansions of some Schrödinger operators", Proc. Amer. Math. Soc. 93 (1985), No. 3, 477-482.

[21] Stempak, K. and Torrea J.L., "On g-functions for Hermite function expansions", Acta Math. Hung. 109 (2005), No. 1-2, 99-125.

[Revista Integración, temas de matemáticas 
[22] Stempak K. and Torrea J.L., "BMO results for operators associated to Hermite expansions", Illinois J. Math. 49 (2005), No. 4, 1111-1132.

[23] Thangavelu S., Lectures on Hermite and Laguerre Expansions, Math. Notes 42, Princeton University Press, Princeton, 1993.

[24] Thangavelu S., "Hermite and special Hermite expansions revisited", Duke Math. J. 94 (1998), No. 2, 257-278.

[25] Thangavelu S., "Multipliers for Hermite expansions", Rev. Mat. Iberoam. 3 (1987), 1-24.

[26] Thangavelu S., "Summability of Hermite expansions I", Trans. Amer. Math. Soc. 314 (1989), No. 1, 119-142.

[27] Thangavelu S., "Summability of Hermite expansions II", Trans. Amer. Math. Soc. 314 (1989), No. 1, 143-170.

[28] Thangavelu S., "Hermite expansions on $\mathbb{R}^{2 n}$ for radial functions", Rev. Mat. Iberoam. 6 (1990), No. 2, 61-73.

Vol. 36, $\left.\mathrm{N}^{\circ} 1,2018\right]$ 